$11 \mid 2016$

Relire L'Acacia

\title{
Claude Simon et le latin : état des recherches
}

Ian De Toffoli

\section{OpenEdition}

Journals

Édition électronique

URL : http://journals.openedition.org/ccs/389

DOI : $10.4000 /$ ccs.389

ISSN : 2558-782X

\section{Éditeur :}

Presses universitaires de Rennes, Association des lecteurs de Claude Simon

\section{Édition imprimée}

Date de publication : 8 juillet 2016

Pagination : 261-264

ISBN : 978-2-7535-4876-3

ISSN : $1774-9425$

Référence électronique

Ian De Toffoli, «Claude Simon et le latin : état des recherches », Cahiers Claude Simon [En ligne], 11 | 2016, mis en ligne le 15 septembre 2017, consulté le 19 avril 2019. URL : http:// journals.openedition.org/ccs/389; DOI : 10.4000/ccs.389 


\title{
CLAUDE SIMON ET LE LATIN : ÉTAT DES RECHERCHES
}

\author{
Ian De TOFFOLI
}

Si les monographies qui analysent la présence du latin (ou de la latinité, voire de l'Antiquité classique ou de la mythologie) dans l'œuvre de Claude Simon sont tout à fait rares - jusqu'à ce jour, il n'y a que le livre de Karen L. Gould ${ }^{1}-$, les articles ou chapitres d'ouvrage qui abordent cette question sont plutôt nombreux.

À l'époque où la recherche simonienne prend de l'ampleur, après les colloques de Cerisy sur le Nouveau roman (1971), puis sur Claude Simon lui-même (1974), quelques travaux abordent le jeu intertextuel latin dans les romans simoniens, c'est-à-dire les mots et les citations latines de César, d'Apulée et de Lucain que l'on retrouve éparpillés dans Histoire ou La Bataille de Pharsale. Notamment Laurent Jenny et, quelques années plus tard, J. A. E. Loubère, dans leurs articles respectifs ${ }^{2}$, donnent à l'intertexte latin une fonction métonymique, métaphorique, métalinguistique ou simplement productrice, c'est-à-dire de stimulus. Michel Bertrand ${ }^{3}$ et Ralph Sarkonak ${ }^{4}$, reprennent et développent ces concepts dans certains chapitres de leurs ouvrages. Pour ce dernier, ainsi que pour des critiques comme

1. Ouvrage un peu daté qui traite exclusivement de l'émergence du mythe et du mythique chez Simon: Karen L. Gould, Claude Simon's mythic muse, Columbia, French Literature Publications, 1979.

2. Laurent Jenny, "La Stratégie de la forme", Poétique, n² 27, 1976, p. 257-281 et J. A. E. Loubère, "The generative function of translation in the novels of Claude Simon ", dans Randi Birn et Karin L. Gould (dir.), Orion Blinded: Essays on Claude Simon, Lewisburg, Londres et Toronto, Bucknell University Press et Associated University Press, 1981, p. 184-199.

3. Michel Bertrand, Langue romanesque et parole scripturale. Essai sur Claude Simon, PUF, 1987.

4. Ralph Sarkonak, Claude Simon: les carrefours du texte, Toronto, Paratexte, 1986. 
Alastair B. Duncan ${ }^{5}$, Perrine Galand ${ }^{6}$ ou, plus récemment, Jean-Yves Laurichesse $^{7}$ et Anne-Yvonne Julien ${ }^{8}$, l'enjeu de l'intertexte latin est plus important que ne le laisse supposer un simple collage citationnel: Simon reprend et adapte des structures narratives antiques, par exemple par procédés réflexifs qui brouillent les niveaux diégétiques, et fait de l'expérience livresque (l'approche charnelle de la langue latine) le sédiment de son écriture. D'autres critiques, comme Jean Kaempfer ${ }^{9}$ qui analyse la présence plus large du latin chez certains écrivains du nouveau roman tels que Simon, Pinget ou Butor, ou encore, avant lui, Mary $\mathrm{Orr}^{10}$ ou encore Claudia Hoffer Gosselin ${ }^{11}$, considèrent que le latin, et plus précisément le texte latin lu et appris pendant sa scolarité ${ }^{12}$, a imprégné l'écrivain qu'est devenu Claude Simon, au point de constituer dans plusieurs romans une pièce clé du dispositif narratif simonien.

D’un autre côté, la critique a remarqué le discrédit infligé à la culture humaniste mis en avant dans certains romans simoniens ${ }^{13}$ : Mary $\operatorname{Orr}^{14}$ notamment, dans sa monographie (qui ne traite pas que du latin), voit dans l'arrangement citationnel latin - tout comme dans les Géorgiques, de façon

5. Alastair B. Duncan, " Notice sur La Bataille de Pharsale », dans Claude Simon, Euvres I, Gallimard, "La Pléiade », 2006, p. 13.

6. Perrine Galand, "Génériques maniéristes dans les romans d'Achille Tatius et Claude Simon ", La Licorne, $\mathrm{n}^{\circ} 35,1995$, p. 247-259.

7. Jean-Yves Laurichesse, «La guerre en latin. Claude Simon et la bataille de Pharsale " dans Corinne Bonnet et Florence Bouchet (dir.), Translatio: traduire et adapter les Anciens, Classiques Garnier, 2013, p. 285-303.

8. Anne-Yvonne Julien, "Claude Simon, le retour aux choses latines mêmes ", Cahiers Claude Simon, $n^{\circ}$ 9, PUR, 2014, p. 97-111.

9. Jean Kaempfer, "Le latin des nouveaux romanciers ", Poétique, n¹13, fév. 1998, p. 45-59 et Poétique du récit de guerre, Corti, 1998.

10. Mary Orr, "Literature in Literature in Claude Simon's La Bataille de Pharsale ", Romance Studies, $\mathrm{n}^{\circ} 12,1988$, p. 65-76.

11. Claudia Hoffer Gosselin, "Voices of the Past in Claude Simon's La Bataille de Pharsale ", dans Jeanne Parisier Plottel and Hanna Charney (dir.), Intertextuality. New Perspectives in Criticism, New York Literary Forum, vol. 2, 1978, p. 23-33.

12. Simon l'évoque dans plusieurs entretiens ou discours; voir "Il n'y a pas d'art réaliste ", entretien avec Madeleine Chapsal, La Quinzaine littéraire, n 41, 15-30 déc. 1967, p. 4-5, et "Roman et mémoire ", Revue des Sciences humaines, n² 220, oct.-déc. 1990, p. 191-192.

13. Jean-Paul Roelens, "Antiquité et Histoire chez Claude Simon ", Cabiers de l'Université de Perpignan, $n^{\circ} 1$ ("Claude Simon»), 1986, p. 101-118; Francine Dugast-Portes, "Vestiges et fragments: l'Antiquité selon Claude Simon ", dans Au miroir de la culture antique. Mélanges offerts au Président René Marache, PUR, 1992, p. 113-134.

14. M. Orr, Claude Simon: the intertextual dimension, Glasgow, University of Glasgow, French and German publications, 1993. 
plus diffuse - une mise à mort des pères littéraires. D'ailleurs, la critique simonienne reste divisée sur ce dernier roman, les uns insistant sur une vague réécriture ironique ou effacée du poème latin ${ }^{15}$, les autres sur de multiples rapprochements et - comme l'a récemment fait Claire Guizard - une transposition sérieuse de l'hypotexte virgilien ${ }^{16}$.

Un dernier point qu'il faut envisager est celui de la mythocritique. Quoique diffus et fragmenté, le mythe est présent dans l'œuvre simonienne et certains critiques lui ont consacré des articles ou des chapitres de leurs ouvrages, se penchant avant tout sur des cas isolés d'émergence mythique dans La Route des Flandres et le mythe d'Orphée dans Les Géorgiques ${ }^{17}$. Plus rares sont les auteurs, comme Karen L. Gould déjà citée, qui offrent une vue globale de la reprise du mythe par Claude Simon, par affleurement thématique (comme l'écrit Dominique Viart ${ }^{18}$ ), ou par mythonymie (terme forgé par Metka Zupančič ${ }^{19}$ ).

Certains chercheurs abordent également la question de la latinité à travers une approche plus socio-littéraire, comme Mireille Calle-Gruber dans sa biographie de Claude Simon ${ }^{20}$ ou Françoise Waquet ${ }^{21}$, qui explique dans

15. A. B. Duncan, "Claude Simon's Les Géorgiques: an intertextual adventure ", Romance Studies, $\mathrm{n}^{\circ} 2$, 1983, p. 90-107; Cora Reitsma-La Brujeere, Passé et présent dans Les Géorgiques de Claude Simon. Étude intertextuelle et narratologique d'une reconstruction de l'Histoire, Amsterdam, Rodopi, 1992; Anne-Marie Monluçon, "Le fantôme des Géorgiques dans Les Géorgiques de Claude Simon ", dans Frontières et passages, PU de Rouen, 1999, p. 331-337, et "L’Orphée de Virgile, ou la face cachée des Géorgiques de Claude Simon ", Cahiers de l'Association internationale des études françaises, 2001, $\mathrm{n}^{\circ} 53$, p. 271-285.

16. Claire Guizard, Claude Simon, la répétition à l'cuvre: Bis repetita, L'Harmattan, 2005; Marie Miguet, "Lecture mythique des Géorgiques de Claude Simon ", dans G. Freyburger (dir.), De Virgile à Jacob Blade. Hommage à $M^{m e}$ Andrée Thill, Bulletin de la Faculté de lettres de Mulhouse, nº 15, 1987, p. $273-$ 284; Nathalie Piégay-Gros, Claude Simon, Les Géorgiques, PUF, 1996; Pierre Schoentjes, Claude Simon par correspondance. Les Géorgiques et le regard des livres, Genève, Droz, 1995.

17. Henri Béhar, "La Culture de l'auteur et la compétence du lecteur dans La Route des Flandres", Littératures contemporaines, $\mathrm{n}^{\circ} 3$, 1997, p. 207-227; Michael J. Evans "The Orpheus myth in Les Géorgiques and Homage to Catalonia ", dans A. B. Duncan (dir.), Claude Simon: New Directions, Edimbourg, Scottish Academic Press, 1985, p. 89-99; N. Piégay-Gros, "Légende et affabulation dans La Route des Flandres", Littératures contemporaines, n 3, 1997, p. 119-131; Catherine Rannoux, L'Écriture du labyrinthe: Claude Simon, La Route des Flandres, Orléans, Paradigme, 1997, p. 131-142; Metka Zupančič, "Érotisme et mythisation dans Triptyque et Les Géorgiques ", La Revue des Lettres modernes, série Claude Simon, n 2 ("L'écriture du féminin-masculin »), 1997, p. 35-54.

18. Dominique Viart, "Mythes et imaginaire des signes: Butor, Robbe-Grillet, Simon ", dans Pierre Cazier (dir.), Mythe et Création, PU de Lille, 1994, p. 267-285.

19. M. Zupančič, Lectures de Claude Simon: la polyphonie de la structure et du mythe, Toronto, Éd. du Gref, 2001 et "De la (dé)nomination à la mythisation chez Claude Simon ", Paragraphes, n 10 (Les noms du roman), Montréal, Département d'Études Françaises, 1994, p. 103-115.

20. Mireille Calle-Gruber, Claude Simon. Une vie à écrire, Le Seuil, 2011.

21. Françoise Waquet, Le Latin ou l'empire d'un signe, (xvi $\mathrm{x}^{\mathrm{e}} \mathrm{xx}^{\mathrm{e}}$ siècle), Albin Michel, 1998. 
son livre la fascination sexuelle des adolescents pour la langue opaque qu'est le latin grâce à des citations tirées d'Histoire.

On voit donc que la présence du latin dans l'œuvre simonienne est loin d'être inexplorée, mais elle ne constitue le plus souvent qu'une perspective secondaire, au sein d'un cadre de réflexion plus large et tout autre, par exemple l'intertextualité, l'étude d'un roman particulier, la représentation d'une période historique ou, tout simplement, un trait stylistique précis. 